\title{
The Value of "Bespoke": Demand Learning, Preference Learning, and Customer Behavior
}

\author{
Tingliang Huang \\ Carroll School of Management, Boston College, Chestnut Hill, Massachusetts 02467, tingliang.huang@bc.edu \\ Chao Liang \\ Cheung Kong Graduate School of Business, Beijing 100738, China, cliang@ckgsb.edu.cn \\ Jingqi Wang \\ Faculty of Business and Economics, The University of Hong Kong, Pokfulam, Hong Kong, jingqi@hku.hk
}

\begin{abstract}
"Bespoke" or mass customization strategy combines demand learning and preference learning. We develop an analytical framework to study the economic value of bespoke systems and investigate the interaction between demand learning and preference learning. We find that it is possible for demand learning and preference learning to be either complements or substitutes, depending on the customization cost and the demand uncertainty profile. They are generally complements when the personalization cost is low and the probability of having high demand is large. Contrary to usual belief, we show that higher demand uncertainty does not necessarily yield more complementarity benefits. Our numerical study shows that the complementarity benefit becomes weaker when customers are more strategic. Interestingly, the substitute loss can occur when the personalization cost is small and the probability of having high demand is large, when customers are strategic.
\end{abstract}

Key words: operations-marketing interface; customization; demand forecast; customer behavior

\section{Introduction}

Burberry, a British luxury fashion firm, recently made its first attempt at mass customization by launching "Burberry Bespoke," a business system that allows consumers to design and purchase their own personalized version of the company's iconic trench coats online (Chowney 2011, Lariviere 2011, Sonne 2011, Cooper 2012). At its core, this bespoke strategy combines at least two salient components: (1) demand (quantity) learning before production, enabling a better matching of costly supply with uncertain demand (see Lariviere 2010), and (2) preference learning that generates highly customized/personalized products improving consumer valuation or satisfaction.

Many firms, including Burberry, adopt the bespoke strategy: Blank Label empowers consumers to design their own dress shirts (Wallace 2010); Timbuk2 offers customized messenger bags online (Lariviere 2011); eShakti exclusively focuses on custom and tailored women's clothing, allowing customers the option of selecting from among the store's ever-changing collection of styles and 
designs; Modern Tailor offers customized menswear and women's shirts; Indochino specializes in customizing dress shirts; and so on. ${ }^{1}$ In this industry, the bespoke business model becomes a reality due to "the dawn of tablets and apps, and the rise of cheaper, more-advanced web technologies" (Chowney 2011). Moreover, 3D printing spurs a manufacturing revolution that makes bespoke significantly cheaper and faster for many firms (Vance 2010).

Bespoke is certainly not used only by fashion firms nor even only in the apparel industry. A variety of firms across different industries use the well-known mass customization system. Indeed, a Google search for "customized products" gives us 140 million results as of May 29, 2016, that include a wide range of products. This suggests that customization is widely adopted by numerous companies in a number of industries far beyond fashion and apparel.

The central question we are interested in is whether the bespoke or mass customization strategy is recommended (in the fashion apparel industry or in other nonfashion industries). In the operations management literature, it is well known that having more demand information helps reduce inventory cost (see, e.g., Wang and Toktay 2008, Özer 2011, Huang and Van Mieghem 2014); there is an extensive body of the marketing literature showing the value of personalization or customization (see, e.g., Murthi and Sarkar 2003, Pal and Rangaswamy 2003, Fay et al. 2009). ${ }^{2}$ At first glance, it may appear obvious that combining these two learning effects together should be a good strategy. However, in this paper, we shall argue that it may not be always so. Like Milgrom and Roberts (1990) and Athey and Schmutzler (1995), Cachon and Swinney (2011, p. 2) claim that "complementary strategies should be adopted simultaneously, whereas substitutable strategies are more likely to be adopted in isolation." The focus of this paper is to investigate whether and when demand learning and preference learning are complementary or substitutable strategies.

Conventional wisdom may claim that demand learning and preference learning should always be complementary. The reason is that demand learning reduces supply-demand mismatches, whereas preference learning reduces mismatches between product attributes and consumer preferences. Reducing one type of mismatch should increase the marginal value of reducing the other, because a customized higher-valued product is more worthwhile and less likely to be "wasted." But we shall show that this reasoning may not always hold. We find that whether bespoke systems can be of significant value intricately and jointly depends on various factors, such as costs involved, demand profile, customer strategic behavior, and correlations between the two learning mechanisms.

\footnotetext{
${ }^{1}$ http://lifehacker.com/5909754/five-best-online-custom-clothing-stores, accessed on March 20, 2015.

${ }^{2}$ Following Murthi and Sarkar (2003), we do not distinguish customization versus personalization, and will use the two terms interchangeably.
} 
In this paper, we build an analytical framework that allows us to examine the economic value of a bespoke system in which customer heterogeneity is explicitly modeled. This framework not only captures demand learning (to reduce mismatches between costly supply and uncertain demand) and preference learning (to reduce mismatches between product characteristics and customer preferences) but also allows us to investigate the effects of various factors (e.g., fixed and marginal costs, strategic/forward-looking customer behavior, learning imperfectness, correlation between the learning mechanisms). Our main contributions to the existing literature include the following:

1. We build an analytical framework for a firm making both operational decisions (e.g., production quantity) and marketing decisions (e.g., pricing, personalization) when selling to a market with uncertain demand quantity and heterogeneous consumers. We establish that this (stylized) framework can be rationalized by (practical) product line design and assortment planning.

2. We show that demand learning and preference learning are generally complements when the marginal cost of personalization/customization is low and the probability of having high demand is large. In particular, higher demand uncertainty does not necessarily yield more complementarity benefits.

3. Numerically, we find that the complementarity benefit between demand learning and preference learning becomes weaker when customers are more strategic. Interestingly, the substitute loss can occur when the personalization cost is small and the probability of having high demand is large, if customers are strategic.

The outline of this paper is as follows. After reviewing related literature in $\S 2$, we present our basic modeling framework and study whether demand learning and preference learning are complements in $\S 3$ and $\S 4$. We consider a series of model extensions in $\S 5$ and conclude in $\S 6$. Proofs are relegated to the Online Supplement.

\section{Literature Review}

This paper is related to several branches of research in operations management, marketing, and economics.

Our study is related to the significant body of literature in both marketing and operations on product differentiation, mass customization, or personalization. Customization is intended to provide superior value to customers by meeting their unique needs for products and services (Pine 1999, Liechty et al. 2001, Wind and Rangaswamy 2001, Syam and Kumar 2006, Çil and Pangburn 2017). The bespoke system deployed by Burberry can be thought of as customization whereby customers design their own coats online. The marketing and operations literature has studied a 
variety of benefits of mass customization: it helps build brand loyalty, fit products to the heterogeneous needs of a market, differentiate the offerings of a manufacturer (Lee and Tang 1997, Wind and Rangaswamy 2001, Randall et al. 2007), and develop the awareness of being the creator of the product design (Franke et al. 2010). However, the demand learning aspect of customization has not received much attention in the marketing literature. In the operations literature, considerable research attention has been paid to operational aspects of customization: make-to-order versus make-to-stock (Dobson and Yano 2002, Alptekinoğlu and Corbett 2010), customization versus proliferation in product-line competition (Mendelson and Parlakturk 2008a), and mass customization versus mass production (Alptekinoğlu and Corbett 2008), to name just a few. The existing literature typically ignores the demand learning part by adopting a model featuring deterministic demand. One of the major contributions of this paper is that we capture the important fact that demand is typically inherently uncertain, yet the bespoke system allows the firm to make a customized/personalized product after a customer submits an order. Although this system is designed to learn demand automatically, we do not ignore the fact that the demand would actually be uncertain for the firm without such a bespoke system. Our paper appears to be among the first to explore the interaction of the demand learning and preference learning components that constitute mass customization or bespoke.

Our study is also related to the significant literature that explicitly considers strategic consumer behavior; see, for example, Cachon and Swinney (2011), Tereyağoğlu and Veeraraghavan (2012), Huang and Van Mieghem (2013), Li et al. (2014), Liang et al. (2014) and Huang and Liu (2015), as well as references therein. We study its implications for the economic value of the bespoke strategy, which is a new addition to this literature. In particular, our study is closely related to and complements the recent work of Tereyağoğlu and Veeraraghavan (2012), who focus on firms that are confronted with precipitous trade-off between high sales volume and high profit margins because of highly uncertain market demand, strategic consumer behavior, and the display of conspicuous consumption. Their research questions are mainly about how firms should make decisions such as how much of a good to produce, how to price goods, and when to source from expensive suppliers. In this paper, we focus on firms that use the bespoke system. Although in our model the firm also has to make its pricing and quantity decisions, our research goal is to better understand the economic value of such a business strategy, and thus to provide useful managerial guidelines regarding when this bespoke business strategy is most valuable. In terms of modeling frameworks, whereas Tereyağoğlu and Veeraraghavan (2012) primarily focus on a newsvendor model with homogeneous consumers, 
we use a Hotelling model to capture consumer heterogeneity (see, e.g., Jerath et al. 2010), an essential feature of the market in which bespoke is adopted.

In terms of the modeling framework, the closest to ours is Cachon and Swinney (2011). However, there are several notable major differences: First, we study different practices across different industries (beyond the fashion apparel industry); they study fast fashion (which is a combination of quick response and "enhanced design"), whereas we investigate bespoke or mass customization. Second, there is a fundamental difference between the "enhanced design" and product customization from preference learning here. In Cachon and Swinney (2011), "enhanced design" is modeled as the firm's capability for value enhancement of the product to homogeneous consumers. In our model, mass customization is the firm's capability of reducing the mismatch between the product and each individual consumer's taste/preference. Thus modeling consumer heterogeneity is essential in our setting, whereas in their model, consumers are homogeneous. Third, our research findings are also different. For example, Cachon and Swinney (2011) find that the complementarity benefit between quick response and enhanced design is stronger when customers are more strategic. In sharp contrast, we show that the complementarity benefit between demand learning and preference learning is weaker when customers become more strategic. This finding complements the existing literature by demonstrating that strategic consumer behavior plays different roles in generating values across distinct business practices. Our paper is also related to Li and Zhang (2012) who study a preorder practice in which a seller accepts consumer orders before the release of a product. They identify a tension between the benefits of advance demand information and price discrimination when facing strategic consumers. The bespoke strategy is also meant to collect advance demand information to reduce demand uncertainty; however, this strategy is also capable of collecting consumer preference/taste information to reduce preference mismatches (which is absent in Li and Zhang 2012). Indeed, the preorder strategy can be treated as a concrete example of our demand learning system.

Despite their different research settings, our modeling framework is related to that of Jerath et al. (2010) in several respects. First, we both adopt a Hotelling model to capture consumer heterogeneity. Second, we both model demand uncertainty using a discrete two-point distribution. In Jerath et al. (2010), capacity is fixed. However, in our model, stocking quantity is a decision variable, because that is where demand learning provides value.

Finally, our paper is related to the extensive literature in product line design. For example, Netessine and Taylor (2007) study vertically differentiated products with deterministic demand based on an economic order quantity (EOQ) model, showing that simultaneous optimization of the product line design and production schedule leads to insights significantly different from those 
found in the literature (which omits either the demand side or the supply side of the equation). Although our modeling framework differs, our paper shares a similar spirit in that we study the value of jointly considering both of the operational and marketing sides in a bespoke system. We also show that our approach of modeling customization can be rationalized by and connected to product line design.

\section{Basic Model}

To study the interrelationship between demand quantity (i.e., how many customers) learning and preference (i.e., what customers like) learning, we investigate four possible business models. A traditional system represents a typical newsvendor firm that faces demand uncertainty, that only produces a standard product, and that sells to heterogeneous consumers/customers.

A demand (quantity) learning system represents a firm that learns demand information only, that does not engage in any preference learning activities, and that sells only standard products. This system resembles many firms (e.g., Gap, Banana Republic) that do not allow customer personalization but that put efforts into improving demand forecasts (see, e.g., Shin and Tunca 2010). Such demand forecasts could come from various methods - traditional offline market surveys, online surveys, and the like. Firms that allow preorders but that do not accept customized design also effectively fall into this category, because preordering eliminates supply-demand mismatches.

A preference learning system represents a firm that learns consumer preference, that does not learn the aggregate demand quantity information, and that delivers products better matched to consumer tastes. This system represents Burberry's approach: customers, even if they do not buy, "can still create a design and share it on Twitter and Facebook" (Chowney 2011). In doing this, the firm can learn customer preference by monitoring what was designed on Twitter and Facebook; however, the total demand remains unknown, because the design itself cannot reveal the aggregate demand information. There are certainly many other firms that devote substantial effort to learning consumer tastes (from social media, and other channels) so as to improve product design. "Conjoint analysis" has long been used by marketers to learn consumer preference and guide product design (see Green and Srinivasan 1978, 1990 for exhaustive reviews). It may involve asking customers to provide rankings or ratings of individual product profiles or descriptions (Liechty et al. 2001). Although such an analysis provides consumer preference information, the aggregate market demand may be still unknown and the newsvendor problem (i.e., supply-demand quantity mismatching) remains.

Finally, a bespoke or mass customization system represents a firm that learns both aggregate demand quantity and consumer preference and that makes personalized products to order. In 
doing this, the firm knows both of the aggregate demand quantity and consumer preference. It well resembles firms such as Burberry and Blank Label, or firms that rely on traditional online market surveys, or make-to-order, to obtain both demand quantity and preference information simultaneously.

To answer our research question, we analyze the four possible business models, respectively. The firm chooses its selling price and capacity/inventory level. Heterogeneous consumers choose whether to buy the product. We first focus on the basic model of a traditional system, in which the firm has neither demand learning nor preference learning capabilities. This model is a benchmark based on which the other three systems will be investigated one by one.

\subsection{The Traditional System}

We use the horizontal product differentiation model (i.e., Hotelling 1929): specifically, heterogeneous consumers are uniformly distributed on a unit interval $\left[-\frac{1}{2}, \frac{1}{2}\right]$ with the firm located in the middle. ${ }^{3}$ This assumption is consistent with the literature; see, e.g., Wang and Shin (2015) and references therein. Consumers have a per-unit traveling cost $t$, which measures the preferencemismatch disutility between the firm's product and consumers' tastes. A consumer's utility is the valuation (of her ideal product) less her traveling cost and the product price. For example, if a consumer located at $\theta \in\left[-\frac{1}{2}, \frac{1}{2}\right]$ buys a product with valuation $w$ (of her ideal product) at price $p$ from the firm, her net utility is $u(\theta, p) \equiv w-p-|\theta| t$. A consumer needs at most one product and makes her purchase decision to maximize her net utility.

The total number of consumers in the market $D$ is random. We assume that with probability $\alpha$, the total number of consumers in the market is $d_{H}$, and with probability $1-\alpha$, the total number of consumers in the market is $d_{L}\left(<d_{H}\right)$, where $\alpha \in(0,1)$. This assumption of a simple two-point demand distribution is made for analytical convenience and is also consistent with the existing literature (see, e.g., Jerath et al. 2010). The firm faces the newsvendor problem with endogenous pricing: to choose both the production quantity $q$ and price $p$ to maximize its expected profit (see Petruzzi and Dada 1999 and references therein). ${ }^{4}$ The firm's per-unit production cost is $c$. We assume that $w \leq c+t$ throughout the paper. This assumption means that if the firm were located at $-\frac{1}{2}$, there would be no profit margin to serve the consumers who prefer the product the least-those who are located at $\frac{1}{2}$. In other words, this assumption allows us to focus on the interesting case in which it is not profitable to simply offer the most unfavorable product for the consumers who

\footnotetext{
${ }^{3}$ One can indeed show that the firm cannot do better by locating anywhere else.

${ }^{4}$ In the Online Supplement, we also analyze a variant of this basic model by allowing the firm to price after seeing the demand realization.
} 
like the product the least in the market. From another perspective, the assumption is equivalent to $t \geq w-c$, which means that the mismatch between the consumer preference and the product $t$ is not too small. This is precisely the setting in which personalization brings value. We denote the expected demand $\mu_{D} \equiv \alpha d_{H}+(1-\alpha) d_{L}$.

We characterize the firm's optimal pricing and production quantity strategies, as well as the optimal expected profits, hereafter.

Proposition 1. In a traditional system:

(i) If $\frac{w}{c} \leq \frac{d_{H}-\sqrt{d_{L} \mu_{D}}}{\mu_{D}-\sqrt{d_{L} \mu_{D}}}$, the firm's optimal price $p_{0}^{*}=p_{L}=\frac{w+c}{2}$, optimal production quantity $q_{0}^{*}=q_{L}=$ $\frac{d_{L}(w-c)}{t}$, and optimal profit $\pi_{0}^{*}=\pi\left(p_{L}, q_{L}\right)=\frac{d_{L}}{2 t}(w-c)^{2}$.

(ii) If $\frac{w}{c} \geq \frac{d_{H}-\sqrt{d_{L} \mu_{D}}}{\mu_{D}-\sqrt{d_{L} \mu_{D}}}$, the firm's optimal price $p_{0}^{*}=p_{H}=\frac{\mu_{D} w+d_{H} c}{2 \mu_{D}}$, optimal production quantity $q_{0}^{*}=q_{H}=\frac{d_{H}\left(\mu_{D} w-d_{H} c\right)}{\mu_{D} t}$, and optimal profit $\pi_{0}^{*}=\pi\left(p_{H}, q_{H}\right)=\frac{\mu_{D}}{2 t}\left(w-\frac{d_{H}}{\mu_{D}} c\right)^{2}$.

Proposition 1 shows that the firm's optimal strategy depends on the value-to-cost ratio $\frac{w}{c}$ and the demand distribution profile measured by $\frac{d_{H}-\sqrt{d_{L} \mu_{D}}}{\mu_{D}-\sqrt{d_{L} \mu_{D}}}$. The intuitive implication is that if the value-to-cost ratio $\frac{w}{c}$ is low, the firm should offer a low price and produce a low quantity to achieve the highest profit. Otherwise, offering a high price and a high quantity is the optimal strategy.

\subsection{The Demand Learning System}

In the demand learning system, a firm strives to learn the uncertain market demand but still produces the standard product. The demand learning can take various forms: preorders (without customization), market surveys (online or offline), and demand forecasting (Shin and Tunca 2010 and references therein) among many others.

Different from the traditional system, the firm now can learn the realization of $D$ perfectly before determining its production quantity $q$. In practice, there is typically a fixed investment/implementation cost for demand learning. However, we do not include such a fixed cost in our basic model, for several reasons: First, this allows us to isolate and focus on the interaction of demand learning and preference learning. Second, it is a good first step to understanding the benefit side (we shall include the cost side in $§ 5.3$ ). Third, it has been shown in the literature that rational customers are actually willing to provide their demand information to the firm for free (see, e.g., Huang and Van Mieghem 2013).

We have the following equilibrium results for the demand learning system.

Proposition 2. In a demand learning system: The firm's optimal price $p_{A}^{*}=p_{L}=\frac{w+c}{2}$, optimal production quantity $q_{A}^{*}(D)=\frac{w-c}{t} D$ for a demand realization $D$ and optimal profit $\pi_{A}^{*}=\frac{\mu_{D}}{2 t}(w-c)^{2}$. 
Demand learning affects firm profit via two distinct effects: (1) The matching effect is generated by reducing both overstock and lost sales-that is the production quantity $q_{A}^{*}(D)$ accurately matches the demand quantity. (2) The price optimization effect come from optimally (weakly) lowering its price to increase the number of consumers who are willing to purchase-that is $p_{A}^{*} \leq p_{0}^{*}$. Recall that in the traditional system, when the value-to-cost ratio $\frac{w}{c}$ is high, the firm has to ask for a high price $p_{0}^{*}=p_{H}=\frac{\mu_{D} w+d_{H} c}{2 \mu_{D}}\left(>p_{A}^{*}=p_{L}=\frac{w+c}{2}\right)$ to gain a high profit margin so as to compensate for the potential overstock cost.

\subsection{The Preference Learning System}

In the preference learning system, the firm uses various channels, techniques, and methods, such as social media (Chowney 2011), clickstream tracking (Murthi and Sarkar 2003), intelligent agents, collaborative filtering and content filtering (Murthi and Sarkar 2003), and conjoint analysis (Green and Srinivasan 1978, 1990, and Terwiesch and Loch 2004), to collect preference information from customers and thus can customize/personalize its product to customers' preference and taste. "Personalized products" create greater benefits for customers than "standard products," because they deliver a closer preference fit (Franke et al. 2009). However, the total market size remains uncertain without demand learning. Hence, the firm still faces the newsvendor problem. We simply model product personalization/customization as the firm's capability to enhance customer valuation upon observing customer preference information by completely removing the preference-mismatch cost $t$. Let $c_{p} \equiv c+\Delta c_{p}(<w)$ be the marginal production cost with personalization/customization, where $\Delta c_{p}>0$.

Proposition 3. In a preference learning system, the firm charges the optimal price $p_{p}^{*}=w$ :

(i) If $w \geq \frac{c_{p}}{\alpha}$, the firm's optimal production quantity $q_{p}^{*}=d_{H}$ and the optimal profit $\pi_{p}^{*}=w \mu_{D}-c_{p} d_{H}$. (ii) If $w<\frac{c_{p}}{\alpha}$, the firm's optimal production quantity $q_{p}^{*}=d_{L}$ and the optimal profit $\pi_{p}^{*}=\left(w-c_{p}\right) d_{L}$.

The explanation of Proposition 3 is that the firm can offer the highest price $w$ to extract all consumer surplus. If the cost of customization is low, it is optimal for the firm to offer the highest quantity of $d_{H}$. Otherwise, offering a low quantity of $d_{L}$ maximizes its expected profit.

Preference learning affects firm profit via three distinct effects: (1) The valuation effect is generated by reducing mismatches between the product and customer preference so that the firm enjoys a higher price. (2) The sales effect comes from the increase in total sales due to customization. (3) The cost effect comes from adding a customization cost $\Delta c_{p}>0$ to the marginal production cost. 


\subsection{The Bespoke System}

The bespoke or mass customization system combines the characteristics of the demand learning and preference learning systems by allowing customers to order and design customized products. As a result, the firm is able to eliminate any supply-demand mismatch and reduce mismatch between product characteristics and consumer tastes simultaneously. ${ }^{5}$ We characterize the firm's optimal strategy as follows.

Proposition 4. In a bespoke system, the firm's optimal price $p_{A P}^{*}=w$, optimal production quantity $q_{A P}^{*}(D)=D$ for a demand realization $D$, and optimal profit $\pi_{A P}^{*}=\left(w-c_{p}\right) \mu_{D}$.

The firm charges the highest price $w$, and every customer in the market would like to buy the product. Furthermore, the firm produces a quantity exactly equal to demand.

In the next section, we shall investigate the interrelationship between demand quantity and preference learning using the outcomes characterized for the four separate systems in this section.

\section{The Interaction of Demand Learning and Preference Learning}

In this section, we investigate the impact of combining demand learning and preference learning in a bespoke system. In particular, we are interested in answering the following question: are demand learning and preference learning complements or substitutes? If they are complements, then using bespoke generates a super-additive benefit: the incremental value of a bespoke system is higher than the combined incremental value of demand learning and preference learning used in isolation - that is $\pi_{A P}^{*}-\pi_{0}^{*} \geq\left(\pi_{A}^{*}-\pi_{0}^{*}\right)+\left(\pi_{p}^{*}-\pi_{0}^{*}\right)$. Thus to determine whether demand learning and preference learning are complementary, we check whether

$$
\pi_{0}^{*}+\pi_{A P}^{*} \geq \pi_{A}^{*}+\pi_{p}^{*}
$$

holds (or, equivalently, $\pi_{A P}^{*}-\pi_{A}^{*} \geq \pi_{p}^{*}-\pi_{0}^{*}$ ).

The first finding is that it is possible for demand learning and preference learning to be either complements or substitutes in general. Recall that demand learning improves firm profit through a matching effect, by reducing overstock and lost sales, and a price optimization effect, by weakly lowering its price (recall $p_{A}^{*} \leq p_{0}^{*}$ ). Preference learning affects firm profit via a valuation effect by reducing mismatches between the product and customer preference; a sales effect, by increasing the total sales; and a cost effect, by adding a customization cost. A priori, it is unclear how these distinct effects interact with each other.

\footnotetext{
${ }^{5}$ Additionally, the bespoke process engages customers in an interactive way, which could also enhance customer satisfaction. Customized products are becoming increasingly popular, as "affluent customers are often looking to invest in a unique experience or product" (Hutzler 2011).
} 
Our goal is to provide analytical conditions under which demand learning and preference learning are complements or substitutes. Let $\kappa(\alpha) \equiv \frac{d_{H}-\sqrt{d_{L} \mu_{D}}}{\mu_{D}-\sqrt{d_{L} \mu_{D}}}$, which is a measure of the demand distribution profile. We thus have the following lemma, which will be useful for characterizing conditions:

Lemma 1. $\kappa(\alpha)$ is decreasing in $\alpha$, and $\frac{1}{\alpha} \leq \kappa(\alpha) \leq \frac{2-\alpha}{\alpha}$.

Based on Lemma 1 , there exists $\alpha_{0}>\frac{c}{w}$ such that $\kappa\left(\alpha_{0}\right)=\frac{w}{c}$. We are now ready to provide the sufficient and necessary conditions for demand learning and preference learning to be complements.

Theorem 1. Demand learning and preference learning are complements if, and only if, one of the following two conditions holds:

(1) $\alpha \leq \alpha_{0}$ and $c_{p} \leq w-\frac{(w-c)^{2}}{2 t}$.

(2) $\alpha \geq \alpha_{0}$ and $c_{p} \leq \bar{C}_{p}(\alpha)$, where $\bar{C}_{p}(\alpha) \equiv \max \left\{w-\frac{c(1-\alpha)}{2 t \alpha}\left[2 w-c\left(1+\frac{d_{H}}{\mu_{D}}\right)\right]\right.$, $\left.\alpha w\right\}$.

In the bespoke system, the firm is able to match supply with demand perfectly, and the average inventory level matches the average demand. By contrast, in the preference learning system, the firm stocks for the low demand if the cost of customization $c_{p}$ is large. Hence, for $c_{p} \geq \alpha w$, as $c_{p}$ increases, the firm's profit decreases more quickly in the bespoke system than in the preference learning system. That is, the benefit of demand learning, in which the firm is already learning the preference, is decreasing in the customization cost for $c_{p} \geq \alpha w$. In addition, the firm's profits in the traditional system and the demand learning system are independent of the customization cost. Thus demand learning and preference learning are complements when $c_{p}$ is small but substitutes when $c_{p}$ is large.

In particular, if the customization cost is sufficiently low, then demand learning and preference learning are complements independent of the demand profile. We state this result as a corollary:

Corollary 1. Demand learning and preference learning are complements if $c_{p} \leq \alpha_{0} w$.

Because $\alpha_{0}>\frac{c}{w}$, according to Corollary 1 , we have the immediate result that if $c_{p}=c$, i.e., if there is no cost effect of customization, then demand learning and preference learning are always complements. This does not depend on the demand distribution profile. When $c_{p}$ is high, demand learning and preference learning are complements only when the probability of having high demand is large. Figure 1 presents a representative example to show that demand learning and preference learning can be either complements or substitutes depending on the probability of having high demand and marginal production cost with customization.

We can analytically show that the cost threshold $\bar{C}_{p}(\alpha)$ increases in the probability of high demand $\alpha$ (as illustrated in Figure 1). One can also show that $\bar{C}_{p}(\alpha)$ also increases in $t$ and $w$. 
Figure 1 The Interrelationship of Demand Learning and Preference Learning $\left(d_{H}=60, d_{L}=10, w=3.0, t=\right.$ $1.9, c=1.2$ )

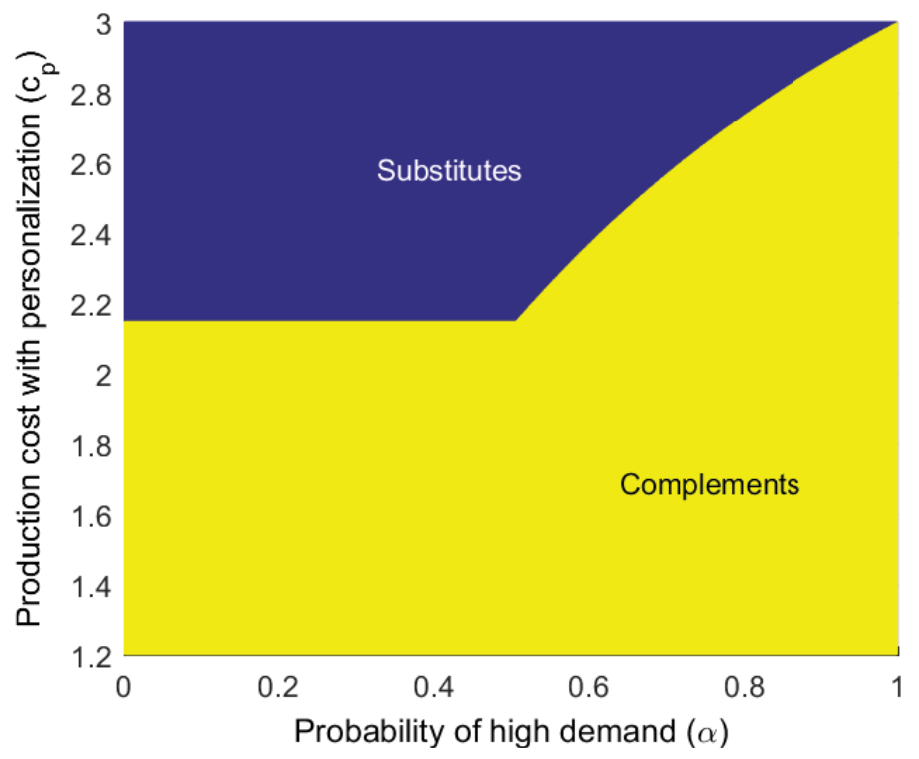

In the traditional system, as long as the percentage of high demand is lower than $\alpha_{0}$, the firm uses a conservative inventory strategy by stocking only for the low demand; thus increasing the percentage of high demand does not really increase the firm's profit. But if $\alpha \geq \alpha_{0}$, then the firm's optimal inventory strategy is to stock for the high demand, and increasing $\alpha$ thus enables the firm to collect more profits in the traditional system. In short, the firm is able to take advantage of a better demand potential (larger $\alpha$ ) only if the demand potential is good enough $\left(\alpha \geq \alpha_{0}\right)$. In such cases, the region where the demand learning and preference learning are complements becomes larger as the probability of having a high demand $\alpha$ increases.

Our results show that preference learning enables the firm to better take advantage of demand learning when the customization cost is low. In addition, when the demand potential exceeds a threshold $\left(\alpha \geq \alpha_{0}\right)$, better demand potential leads to a better synergy between preference learning and demand learning in that they can be complements for a higher customization cost.

We are now interested in the effect of demand uncertainty. Intuitively, one might expect that demand learning and preference learning become complements when demand uncertainty (measured by coefficient of variation) becomes high. However, this is not necessarily the case. Note that the coefficient of variation of the demand is $C V(\alpha) \equiv \frac{\sigma_{D}}{\mu_{D}}=\frac{\sqrt{\alpha(1-\alpha)}\left(d_{H}-d_{L}\right)}{\alpha d_{H}+(1-\alpha) d_{L}}$. We can show that $C V(\alpha)$ first increases and then decreases in $\alpha \in[0,1]$ and reaches its maximum at $\alpha=\frac{d_{L}}{d_{H}+d_{L}}$. To investigate the effect of demand uncertainty, we can vary the magnitude of demand uncertainty 
measured by $C V$ (while keeping other parameters unchanged) and investigate how the threshold $\bar{C}_{p}$ changes. Hence, when treating the threshold $\bar{C}_{p}(C V)$ as a function of the coefficient of variation $C V$, it is not generally monotonic with respect to the coefficient of variation $C V$. That means that higher levels of demand uncertainty do not necessarily yield more complementarity benefits. It is the probability of having high demand, rather than the magnitude of demand uncertainty, that critically determines the interrelationship of demand learning and preference learning. The managerial insight is that the bespoke system does not necessarily work best in settings in which the market demand is highly uncertain.

\section{Extensions}

In the basic model, we made several assumptions: perfect demand learning, perfect preference learning, no fixed investment/implementation costs, no strategic consumer behavior, and no correlation between the two learning mechanisms. In this section, we consider a series of extensions to the basic model to understand what the effects of these factors are.

\subsection{Imperfect Demand Learning}

Suppose that the firm may not learn the market demand quantity perfectly. In the basic model, we assumed that the firm can observe the demand perfectly before making its production decisions. However, in reality, the demand learning may be imperfect. We adopt a Bayesian framework to model imperfect demand learning. Before making its production quantity decision, the firm can observe a signal $s$ that is correlated with the true demand $D$. Let $\beta_{i} \equiv P\left(s=d_{i} \mid D=d_{i}\right)$ denote the probability that the signal correctly indicates demand realization $d_{i}$ when the true demand realization is $d_{i}$, for $i=H, L$. If the signal is perfect, we have $\beta_{i}=1$ for $i=H, L$. If the signal is completely useless, we have $\beta_{H}=\alpha$ and $\beta_{L}=1-\alpha$, in which case there is no demand learning, because the firm can earn the same expected profit by ignoring the signal $s$. Thus for a noisy signal $s$, we have the bound $\beta_{H} \in[\alpha, 1]$ and $\beta_{L} \in[1-\alpha, 1]$. The unbiasedness condition implies

$$
P\left(s=d_{H}\right)=P\left(D=d_{H}\right) P\left(s=d_{H} \mid D=d_{H}\right)+P\left(D=d_{L}\right) P\left(s=d_{H} \mid D=d_{L}\right)=\alpha
$$

and

$$
P\left(s=d_{L}\right)=P\left(D=d_{H}\right) P\left(s=d_{L} \mid D=d_{H}\right)+P\left(D=d_{L}\right) P\left(s=d_{L} \mid D=d_{L}\right)=1-\alpha .
$$

Combining with the total probability rule $P\left(s=d_{j} \mid D=d_{i}\right)=1-P\left(s=d_{i} \mid D=d_{i}\right)$ for $j \neq i$ and $i, j=H, L$, we obtain the equation

$$
\beta_{L}=1-\frac{\alpha\left(1-\beta_{H}\right)}{1-\alpha}
$$


which dictates the one-to-one mapping between $\beta_{H}$ and $\beta_{L}$. Firm's demand learning accuracy, $\gamma$, can then be defined as the expected probability that the signal correctly predicts the demand realization, i.e.,

$$
\gamma \equiv \alpha \beta_{H}+(1-\alpha) \beta_{L}
$$

When demand learning is perfect, i.e., $\beta_{L}=1$, we have the upper bound $\gamma_{\max } \equiv 1$. When demand learning is completely useless, i.e., $\beta_{L}=1-\alpha$, we have $\gamma_{\min } \equiv 2 \alpha^{2}-2 \alpha+1$. To measure the effectiveness/accuracy of the firm's demand learning capability, we define the firm's demand learning accuracy, $I$, by rescaling $\gamma$ upon $[0,1]$ with the following identity:

$$
I \equiv \frac{\gamma-\gamma_{\min }}{\gamma_{\max }-\gamma_{\min }}
$$

Then $I$ is an index measure for the firm's capability to learn demand. When $I=0$, the demand information $s$ is completely useless, so that $\gamma=\gamma_{\min }$. When $I=1$, the demand information $s$ is perfect, so that $\gamma=\gamma_{\max }$.

For analytical tractability yet without losing the qualitative insights, we shall normalize the low demand quantity to be zero in this section, i.e., $d_{L}=0$. The analysis for the traditional system is the same as in the basic model. For the (imperfect) demand learning system, the sequence of events is as follows: The firm can observe the advance demand information $s$ before making its production decision. Based on the realization of $s$, the firm has a posterior demand distribution. Based on this updated demand distribution, the firm solves its newsvendor problem. For expositional convenience, we introduce the notations $I_{1}(\alpha) \equiv 1-\frac{w-c}{(1-\alpha) w}$ and $I_{2}(\alpha) \equiv \frac{\alpha w-c}{\alpha w}$. We characterize the firm's expected profit in the following lemma:

LEMMA 2. In an imperfect demand learning system with accuracy $I$ :

(i) If $\alpha \in\left[0, \frac{c}{w}\right]$ and $I \in\left[I_{1}(\alpha), 1\right]$, the firm optimal expected profit $\pi_{A}^{*}(I, \alpha)=\frac{\alpha(1-(1-I)(1-\alpha)) d_{H}}{2 t}(w-$ $\left.\frac{c}{1-(1-I)(1-\alpha)}\right)^{2}$.

(ii) If $\alpha \in\left[0, \frac{c}{w}\right]$ and $I \in\left[0, I_{1}(\alpha)\right]$, the firm optimal expected profit $\pi_{A}^{*}(I, \alpha)=0$.

(iii) If $\alpha \in\left[\frac{c}{w}, 1\right]$ and $I \in\left[I_{2}(\alpha), 1\right]$, the firm optimal expected profit $\pi_{A}^{*}(I, \alpha)=\frac{\alpha(1-(1-I)(1-\alpha)) d_{H}}{2 t}(w-$ $\left.\frac{c}{1-(1-I)(1-\alpha)}\right)^{2}$.

(iv) If $\alpha \in\left[\frac{c}{w}, 1\right]$ and $I \in\left[0, I_{2}(\alpha)\right]$, the firm optimal expected profit $\pi_{A}^{*}(I, \alpha)=\alpha \frac{(1-(1-I)(1-\alpha)) d_{H}}{2 t}(w-$ $\left.\frac{c}{1-(1-I)(1-\alpha)}\right)^{2}+(1-\alpha) \frac{(1-I) \alpha d_{H}}{2 t}\left(w-\frac{c}{(1-I) \alpha}\right)^{2}$.

The preference learning system and the bespoke system are the same as in the basic model.

We have analytically characterized when imperfect demand learning and preference learning are complements or substitutes and have found that it is still the probability of having high demand and 
the personalization cost that determines the interrelationship between imperfect demand learning and preference learning. However, the demand learning accuracy $I$ also plays a critical role. For exposition convenience, we define $f(I) \equiv \frac{\alpha(1-(1-I)(1-\alpha))}{2 t(1-\alpha)}\left(w-\frac{c}{1-(1-I)(1-\alpha)}\right)^{2}-\frac{\alpha}{2 t(1-\alpha)}\left(w-\frac{c}{\alpha}\right)^{2}$. We can show that there exists $\underline{I}(\alpha) \in(0,1)$ such that $f(\underline{I}(\alpha))=c$. The theorem below shows the robustness of our main result in the basic model to the accuracy of imperfect demand information.

THEOREM 2. (Impact of Imperfect Demand Information) Suppose $I \in\left[\max \left(\underline{I}(\alpha), I_{2}(\alpha)\right), 1\right]$. Imperfect demand learning and preference learning are complements if, and only if, one of the following conditions holds:

(1) $\alpha \leq \frac{c}{w}$ and $c_{p} \leq w-\frac{1-(1-I)(1-\alpha)}{2 t}\left(w-\frac{c}{1-(1-I)(1-\alpha)}\right)^{2}$.

(2) $\alpha>\frac{c}{w}$ and $c_{p} \leq \bar{C}_{p}(I, \alpha)$, where

$$
\bar{C}_{p}(I, \alpha) \equiv \max \left\{w-\frac{1-(1-I)(1-\alpha)}{2 t}\left(w-\frac{c}{1-(1-I)(1-\alpha)}\right)^{2}+\frac{1}{2 t}\left(w-\frac{c}{\alpha}\right)^{2}, \alpha w\right\} .
$$

Interestingly, the sufficient and necessary conditions for complementarity remain structurally the same as long as the demand learning accuracy $I$ is high. We also present some representative numerical examples to show our analytical results in Figure 2. The following notable observations are in order. First, the threshold for the personalization cost now depends on the probability of having high demand $\alpha$ even when $\alpha$ is low. This is different from that in the basic model, in which the threshold for the personalization cost is independent of $\alpha$ when $\alpha \leq \alpha_{0}$. Second, imperfect demand learning enlarges the region in which the bespoke system generates complementarity benefit. The reason is that although the demand learning system in isolation is imperfect (because it typically collects demand information before seeing actual orders), the bespoke system is still perfect in learning demand because production occurs after receiving orders. ${ }^{6}$

\subsection{Imperfect Preference Learning}

In the basic model, we assumed that preference learning is perfect, i.e., the firm can reduce the preference mismatch cost $t$ to zero. However, this might not be the case in practice. Owing to capability limitations or cost considerations of preference learning and customization, the firm may only be able to reduce $t$ to $t_{p} \in(0, t)$ with the production cost $c_{p}(J)=c+\Delta c_{p}(J)$, where $J \equiv \frac{t-t_{p}}{t}=$ $1-\frac{t_{p}}{t} \in(0,1)$ measures the level of customization. A higher $J$ means a better match of consumer

\footnotetext{
${ }^{6}$ One may argue that the Bespoke system may learn demand imperfectly due to reasons such as order cancellations. However, this imperfectness is typically negligible in practice when compared to the imperfectness in the demand learning system. Nevertheless, we have analyzed a setting in which the bespoke system has accuracy $I$ as well. The detailed analysis is available from the authors.
} 
Figure 2 The Impact of the Accuracy of Imperfect Demand Information on the Interrelationship of Demand Learning and Preference Learning $\left(w=3.0, t=1.3, c=2.0, d_{H}=20\right)$
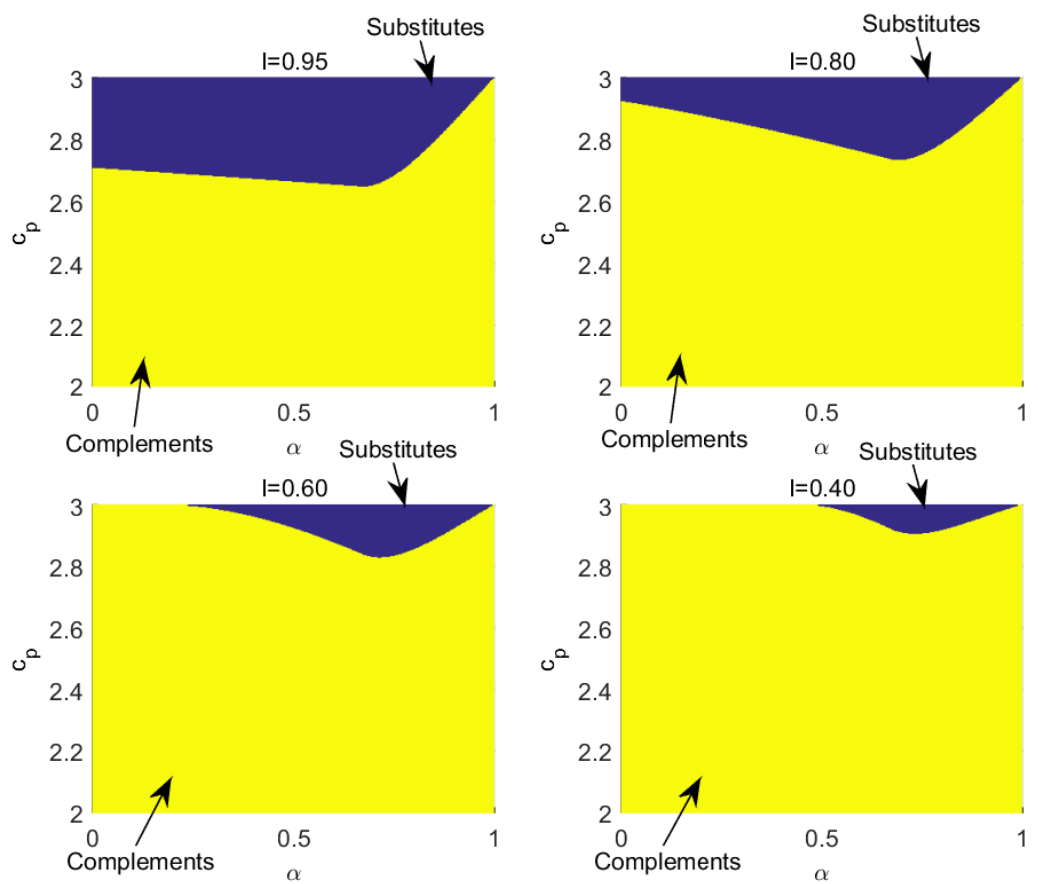

preference and more accurate preference learning. The special case when $J=1$ corresponds to perfect preference learning analyzed in the basic model. We will abuse notation by writing $c_{p}=$ $c_{p}(J)$ given that $J$ is fixed. For analytical convenience, we normalize $d_{L}$ to zero in this section.

REMARK 1. Although our model is a stylized economics model, it can actually be operationalized and supported by the interpretation of product line design or assortment planning. In the Online Supplement, we show that the level of customization $J$ can be equivalently mapped to the number of offered products in the product line; see Netessine and Taylor (2007) for the product line design and Gaur and Honhon (2006) for the assortment planning literatures. We adopt this model also because of its parsimony and tractability.

We first analyze the preference learning system. Next, we analyze the bespoke system where preference learning is imperfect with the level of customization $J$ and demand learning is perfect. The detailed analysis is relegated to the Online Supplement. We are able to provide simple sufficient conditions for demand learning and imperfect preference learning to be complements in Theorem $3:$

THEOREM 3. (Impact of Imperfect Preference Information) Demand learning and imperfect preference learning are complements if one of the following two conditions holds: 
Figure 3 The Impact of the Accuracy of Imperfect Preference Information on the Interrelationship of Demand Learning and Preference Learning $\left(w=7.0, t=5.5, c=5.0, d_{H}=10\right)$
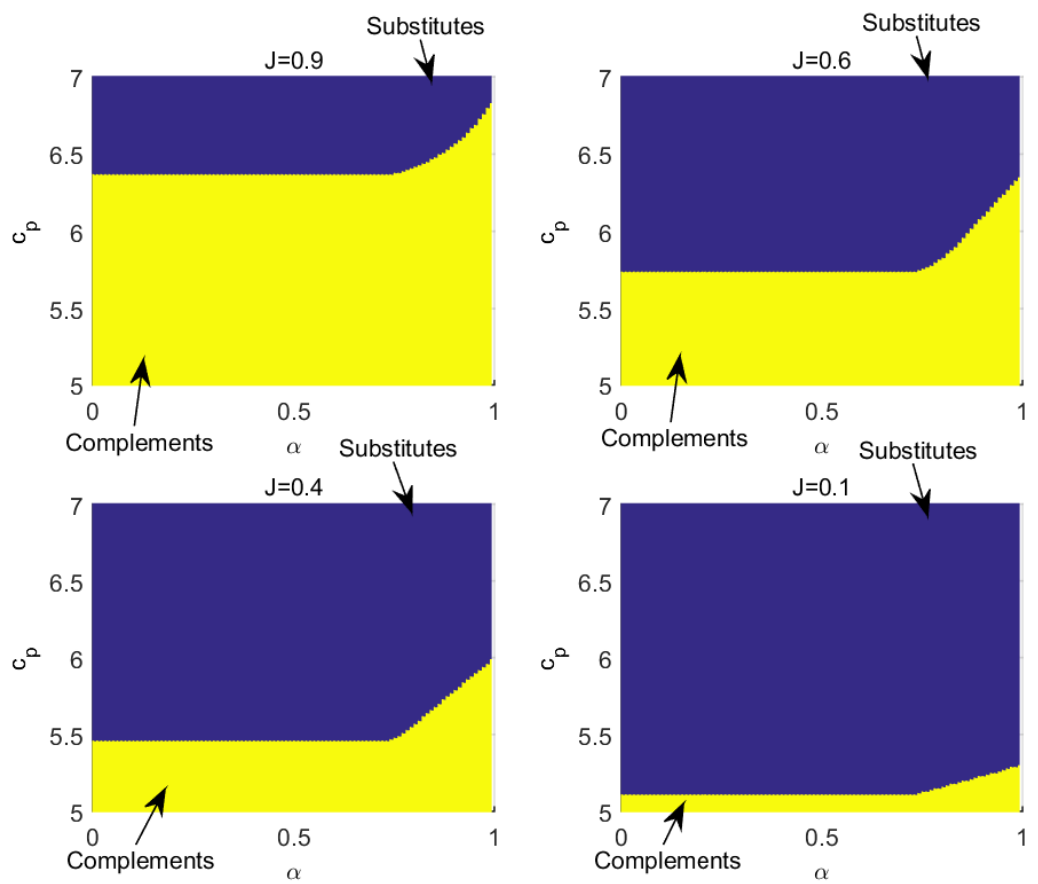

(1) $\alpha \leq \frac{c}{w}$ and $c_{p} \leq \min \left\{w-\frac{(w-c)^{2}}{2 t}-\frac{1}{2} t(1-J), w-\sqrt{1-J}(w-c)\right\}$.

(2) $\alpha>\frac{c}{w}, \alpha \leq \frac{c_{p}}{c_{p}+t(1-J)}$, and $c_{p} \leq \bar{C}_{p}(\alpha, J)$, where

$$
\bar{C}_{p}(\alpha, J) \equiv \max \left\{w-\frac{c(1-\alpha)}{2 t \alpha}\left[2 w-c\left(1+\frac{1}{\alpha}\right)\right]-\frac{1}{2} t(1-J), \alpha w-\alpha t(1-J)\right\} .
$$

Theorem 3 shows a structurally similar condition for complementarity as in the basic model. We again resort to a numerical study to see the impact of imperfect preference learning. Figure 3 shows a set of representative examples, which shows that for any accuracy level $J$, the regions for complements and substitutes are structurally similar to those in the basic model. However, as preference learning becomes less accurate, the region for being complements shrinks.

\subsection{Investment Costs and Endogenous Learning Levels}

In the basic model, we assumed that demand learning and preference learning are perfect. In $§ 5.1-$ 5.2 , we extended the basic model to the case in which demand learning and preference learning are imperfect and the demand learning (accuracy) level $I$ and preference learning/customization (accuracy) level $J$ are exogenous, which applies to settings in which the firm does not have much control over the learning accuracy levels (for example, because of capability or technology constraints or because of purchasing/outsourcing from a third party such as a consulting or market-research 
firm). In this section, we investigate the setting in which the learning accuracy levels $I$ and $J$ are endogenously determined by the firm (e.g., through its own market research and forecasting efforts) and there are investment/implementation costs that are increasing in the learning accuracy levels.

Consistent with the literature (cf. Mendelson and Parlakturk 2008b, Shin and Tunca 2010), we assume that the costs are (weakly) convex in the learning accuracy levels. We have investigated two concrete forms of the learning investment/implementation costs: linear and quadratic. For brevity and without losing our main managerial insights, we present only the case of linear functions in which the investment costs for demand learning, preference learning, and bespoke systems are $C A(I)=K_{A 0}+K_{A}\left(I-I_{0}\right), C P\left(J_{P}\right)=K_{P 0}+K_{P}\left(J_{P}-J_{P 0}\right)$ and $C A P\left(J_{A P}\right)=K_{A P 0}+K_{A P}\left(J_{A P}-\right.$ $\left.J_{A P 0}\right)$ respectively. ${ }^{7}$ The firm spends a fixed $\operatorname{cost} K_{A 0}$ to set up the demand learning system that brings the minimum/basic demand learning level $I_{0}$, and it can increase the learning level at a variable learning cost $K_{A}\left(I-I_{0}\right)$ with a linear function. We have similar explanations for preference learning and bespoke systems. ${ }^{8}$ We have $I_{0} \leq I \leq 1, J_{P 0} \leq J_{P} \leq 1, J_{A P 0} \leq J_{A P} \leq 1, c_{p}>c$, $I_{0} \geq 0, J_{P 0} \geq 0, J_{A P 0} \geq 0, K_{i}>0$, where $i=A, P, A P$, and $K_{j} \geq 0$, where $j=A 0, P 0, A P 0 .{ }^{9}$ The firm chooses the learning accuracy levels $\left(I, J_{P} \text { and } J_{A P}\right)^{10}$ and learns the demand and/or preference before determining the price and production/stocking quantity. For analytical tractability, we normalize $d_{L}$ to zero in this section.

We have analyzed all the three systems and are able to provide full analytical conditions for demand and preference learnings' being complements. We relegate these analytical results to Online Supplement because of their technicality and length but summarize the main qualitative findings hereafter, with representative numerical examples. Much as in the basic model, whether demand learning and preference learning are complements crucially depends on the probability of having high demand $\alpha$ and the marginal production cost with customization $c_{p}$. Generally, they are more likely to be complements when $c_{p}$ is small and the probability of having high demand is large, which is consistent with our basic model. Nevertheless, with endogenous learning levels, the corresponding

${ }^{7}$ The analysis for the quadratic case is available from the authors.

${ }^{8}$ We model $K_{A 0}, K_{P 0}$ and $K_{A P 0}$ as sunk costs as typically done in the existing literature (see, e.g., Mendelson and Parlakturk 2008b, Shin and Tunca 2010). This also allows us to focus on whether and when demand learning and preference learning are complements conditional on that these two kinds of learning are indeed present (i.e., these systems are already set up).

${ }^{9}$ To focus on the role of endogenous learning levels, we assume that the marginal production cost $c_{p}$ with preference learning is independent of the learning level in both preference learning and Bespoke systems, which is consistent with the existing literature (see, e.g., Mendelson and Parlakturk 2008b and references therein). The assumption that a higher customization level entails higher investment costs and does not affect the firm's marginal production cost is justified by the mass customization's premise of achieving mass-production efficiency (Mendelson and Parlakturk 2008b).

${ }^{10}$ For brevity, we will omit the subscripts $P$ and $A P$ for $J$ whenever no confusion arises. 
Figure 4 The Impact of Variable Investment Costs $\left(d_{H}=20, d_{L}=0, w=3.0, t=1.8, c=1.8, K_{A 0}+K_{P 0}=K_{A P 0}\right)$
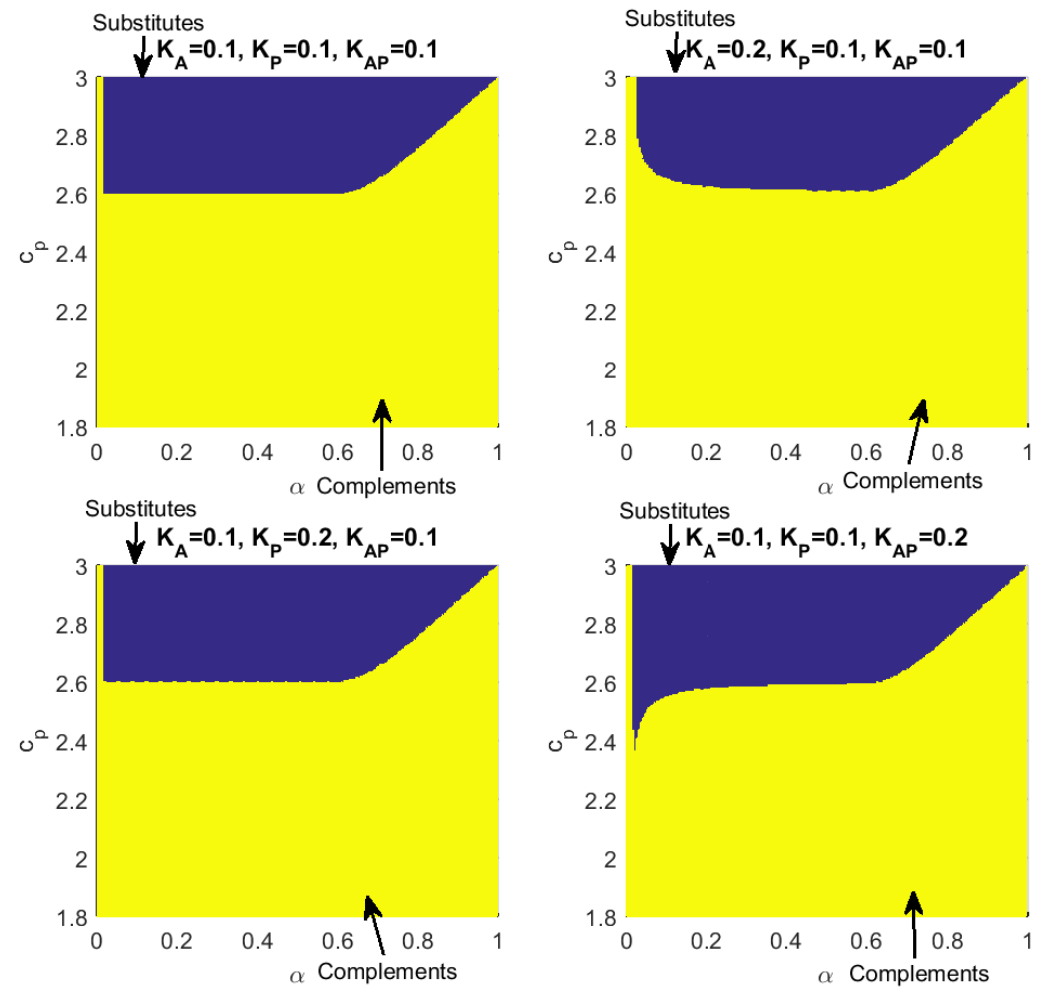

variable investment costs also play an important role. Demand learning and preference learning are more likely to be complements when the variable investment cost of a bespoke system is not very high compared to those of demand learning and preference learning systems. Figure 4 shows the interrelationship between demand learning and preference learning when $K_{A P 0}=K_{A 0}+K_{P 0}$, as the variable investment costs $K_{i}, i=A, P, A P$, vary.

As shown in Figure 4, we observe the interesting result that when $\alpha$ is very small, demand learning and preference learning can always be complements regardless of the value of the marginal production cost with customization $c_{p}$. This result is general and is analytically shown in Proposition 5:

Proposition 5. Suppose that $K_{A P 0} \leq K_{A 0}+K_{P 0}$ and $I_{0} \leq \frac{c}{w}$. There exists $\tilde{\alpha} \equiv \min \{1-$ $\left.\frac{w-c}{\left(1-I_{0}\right) w}, \frac{2 t K_{A}\left(1-I_{0}\right)}{d_{H}(w-c)^{2}}\right\}$ such that demand learning and preference learning are complements for $\alpha \leq \tilde{\alpha}$.

Intuitively, a small probability of having high demand implies a low expected demand, so the firm does not have incentives to improve any of the learning levels owing to the high average learning cost per-unit of the product. Anticipating that the demand realization is probably going to be low, 
the firm prefers a low stocking quantity catering to a low demand realization in both traditional and preference learning systems. In a demand learning system, when the basic learning accuracy level is sufficiently low, demand forecast is not sufficiently accurate so the firm, again, prefers to have a low stocking level. However, the firm knows the demand and perfectly matches it with supply in the bespoke system. Therefore, as long as the fixed cost of the bespoke system is not higher than the sum of those in demand learning and preference learning systems (i.e., $K_{A P 0} \leq K_{A 0}+K_{P 0}$ ), demand learning and preference learning are complements.

The result in Proposition 5 appears different from those of the basic model. The reason is that in the range of small probabilities of having high demand (as shown in Figure 4), the firm does not have incentives to improve the learning accuracy in either demand learning or preference learning systems, while in the basic model with exogenous learning levels (perfect) demand and preference information is already available. Indeed, conditioning on that the firm invests in improving the learning accuracy in both demand and preference learning systems (i.e., in the subrange where the probability of having high demand is not too small), the result, as illustrated in Figure 4, is structurally similar to that of the basic model. This reveals that the bespoke system has an additional advantage if the learning levels are endogenous, because the firm may not have incentives to invest in improving learning accuracy in demand learning and preference learning systems while it learns demand automatically in the bespoke system.

We also investigate numerically how the fixed costs $K_{j}, j=A 0, P 0, A P 0$ affect the interrelationship between demand learning and preference learning. First, note that when $K_{A P 0}=K_{A 0}+K_{P 0}$, we have the same results as if there were no such fixed costs (i.e., $K_{A P 0}=K_{A 0}=K_{P 0}=0$ ). Second, when $K_{A P 0}<K_{A 0}+K_{P 0}$, i.e., learning both demand and preference information is more efficient than learning them in isolation, we have the same structural results as illustrated in Figure 4 although they are more likely to be complements. When the fixed cost of a bespoke system is higher than the sum of those of demand learning and preference learning systems (i.e., $K_{A P 0}>K_{A 0}+K_{P 0}$ ), demand learning and preference learning are more likely to be substitutes as the fixed cost of the bespoke system is higher. Interestingly, these two learning mechanisms can be substitutes when $\alpha$ is very large, because the high fixed cost of bespoke $K_{A P 0}$ directly outweighs the complementarity benefit between the two learning mechanisms when the probability of high demand is too large.

\subsection{Strategic Customer Behavior}

In the basic model, we assume that customers do not consider purchase opportunity in the future and that there is no markdown season or salvage market. In this section, we relax these assump- 
tions. ${ }^{11}$ We now have two selling periods: the first period is for the regular selling season, after which any leftover inventory can be sold in the second period, when markdown pricing may happen. Hence the firm has the flexibility to conduct dynamic pricing in two periods to extract consumer surplus. Customers might exhibit strategic or forward-looking behavior. For example, Rozhon (2004) and Cachon and Swinney (2011) show that in the fashion industry, the propensity of consumers to strategically wait for future markdowns is a well-documented and widespread phenomenon. To account for the degree of strategic customer behavior, we use a discount factor $r$ to measure how strategic/forward-looking customers are. Specifically, in the second period, a strategic customer's valuation of the product is $r w$. A greater $r$ means a more strategic customer population. We acknowledge that there are various factors influencing this discount factor, which might be related to customers' preference of time, intrinsic customer characteristics, product characteristics, and overall market or industry conditions. We assume that the product cost is higher than strategic customers' valuation of the product in the sales period, that is, $c \geq r w$. For $r \in[0, c / w]$, customers are strategic in degree $r$.

Each unsold unit at the end of the second period can be salvaged at value $s$, where $s \in[0, r w]$. In practice, other than selling to the population of strategic customers, the firm may have access to another consumer population such as bargain-hunting customers who purchase the product only when it is on sale. These customers do not even consider purchasing the product in the full-price (regular selling) period (see Cachon and Swinney 2011 and references therein). Consistent with the literature, we assume that bargain-hunting customers' valuation of the product is $s$ and that there are infinitely many such customers in the market at the end of the markdown period. Effectively, those bargain-hunting customers provide an outlet for the firm to dispose/salvage any leftover inventory that cannot be sold to strategic customers. The special case with $r=0$ and $s=0$ becomes our basic model, in which customers are myopic and the salvage value of the unsold inventory is zero (e.g., when there are no bargain-hunting consumers).

With strategic customers, the timeline of the game is as follows:

1. The firm decides the first-period (regular-selling) price $p_{1}$ and the initial stocking/inventory level $q$.

${ }^{11}$ Burberry does have markdowns, for example, at Burberry outlet stores, where the prices are significantly lower than normal. In London, as an anecdotal example, Burberry has one outlet store in east London (while its flagship store is in busy Oxford street in central London). Even in the luxury fashion industry in general, there is evidence that some consumers wait for a good deal. Firms such as bluefly.com and Gilt Groupe specialize in salvage markets for luxury goods (Rice 2010, Tereyağoğlu and Veeraraghavan 2012). As we shall discuss, those consumers can either be strategic consumers or bargain-hunting consumers. Our model allows for the existence of bargain-hunting consumers. 
2. Customers observe the price $p_{1}$ but not the inventory level $q$. They make purchasing decisions based on their rational expectations about the firm's inventory level, denoted as $\bar{q}$. Customer decisions can be summarized into one purchasing threshold $\theta_{1}$. That is, a customer located at $\theta$ buys the product if and only if $|\theta| \leq \theta_{1}$. (This result will be shown in the proof of Proposition 3.)

3. The demand uncertainty and the first period sales are realized.

4. Based on the remaining inventory $q_{2}$ and customers' decisions in period 1 , summarized by $\theta_{1}$, as well as the realized demand, the firm decides the selling price in the second period $p_{2}$.

5. Observing $p_{2}$, customers who did not buy the product in the first period make their purchasing decisions in period 2 , which can be summarized into another threshold $\theta_{2}$. That is, a customer located at $\theta$ buys the product if and only if $\theta_{1}<|\theta| \leq \theta_{2}$. Any leftover inventory at the end of period 2 is cleared at salvage value $s$ per unit.

We first analyze the traditional system, and have the following lemma:

Lemma 3. In the traditional system with strategic customers, the firm's optimal profit can be obtained by optimizing its profit $\pi\left(p_{1}\right)$ with respect to its first-period price $p_{1}$ :

(i) If $\frac{c-(1-\alpha) s}{\alpha} \leq w-r w+s$, then $\pi\left(p_{1}\right)=\left\{\begin{array}{l}2\left(\alpha p_{1}-c\right) \frac{w-p_{1}}{t} d_{L}+2(1-\alpha) p_{1} \frac{w-p_{1}}{t} d_{L} \text { if } p_{1}<\frac{c-(1-\alpha) s}{\alpha}, \\ 2\left(\alpha p_{1}-c\right) \frac{w-p_{1}}{t} d_{H}+2(1-\alpha) p_{1} \frac{w-p_{1}}{t} d_{L}+2(1-\alpha) s \frac{w-p_{1}}{t}\left(d_{H}-d_{L}\right) \quad \text { if } \frac{c-(1-\alpha) s}{\alpha} \leq p_{1}<w-r w+s, \\ \pi^{4}\left(p_{1}\right) \quad \text { if } p_{1} \geq w-r w+s,\end{array}\right.$ where $\pi^{4}\left(p_{1}\right)$ can be obtained by substituting $q=2 d_{H} \frac{2 w-(1-\alpha) r w-2 p_{1}+(1-\alpha) s}{(1+\alpha) t}$ and $\theta_{1}=$ $\frac{2 w-(1-\alpha) r w-2 p_{1}+(1-\alpha) s}{(1+\alpha) t}$ into $\alpha p_{1} q+(1-\alpha)\left(2 p_{1} \theta_{1} d_{L}+\frac{d_{L}\left(r w-t \theta_{1}-s\right)^{2}}{2 t}\right)-c q+(1-\alpha) s\left(q-2 \theta_{1} d_{L}\right)$.

(ii) If $\frac{c-(1-\alpha) s}{\alpha}>w-r w+s$, then

$\pi\left(p_{1}\right)= \begin{cases}2\left(\alpha p_{1}-c\right) \frac{w-p_{1}}{t} d_{L}+2(1-\alpha) p_{1} \frac{w-p_{1}}{t} d_{L} & \text { if } p_{1}<c+(1-\alpha)(1-r) w, \\ \pi^{3}\left(p_{1}\right) & \text { if } c+(1-\alpha)(1-r) w \leq p_{1}<\frac{c-(1-\alpha) s}{\alpha}, \\ \pi^{4}\left(p_{1}\right) & \text { if } p_{1} \geq \frac{c-(1-\alpha) s}{\alpha},\end{cases}$

where $\pi^{3}\left(p_{1}\right)$ can be obtained by substituting $q=2 d_{L} \frac{(1-\alpha)(1+\alpha r) w+\left(\alpha^{2}+\alpha-1\right) p_{1}-\alpha c}{(1-\alpha)(1+\alpha) t}$ and $\theta_{1}=$ $\frac{2 w-(1-\alpha) r w-(2+\alpha) p_{1}+c}{(1+\alpha) t}$ into $\alpha p_{1} q+(1-\alpha)\left(2 p_{1} \theta_{1} d_{L}+\left(q-2 \theta_{1} d_{L}\right)\left(r w-t \theta_{1}-\frac{t\left(q-2 \theta_{1} d_{L}\right)}{2 d_{L}}\right)\right)-c q$.

We next analyze the preference learning system and have the following lemma:

LEMMA 4. In a preference learning system with strategic customers,

1. when $c_{p} \leq \alpha w+(1-\alpha) s$, the optimal stocking quantity is $q^{*}=d_{H}$ and the optimal profit is $\pi^{*}=\alpha w d_{H}+(1-\alpha)\left(w d_{L}+s\left(d_{H}-d_{L}\right)\right)-c_{p} d_{H} ;$

2. when $c_{p}>\alpha w+(1-\alpha) s$, the optimal stocking quantity is $q^{*}=d_{L}$ and the optimal profit is $\pi^{*}=w d_{L}-c_{p} d_{L}$.

In the demand learning and bespoke systems, the firm knows the demand realization. Because $c \geq$ $r w$, the firm will not sell in the second period. Thus the optimal strategy and profit are the same as 
Figure 5 The Impact of Strategic Customer Behavior $\left(d_{H}=60, d_{L}=10, w=3.6, t=1.8, c=1.8\right)$
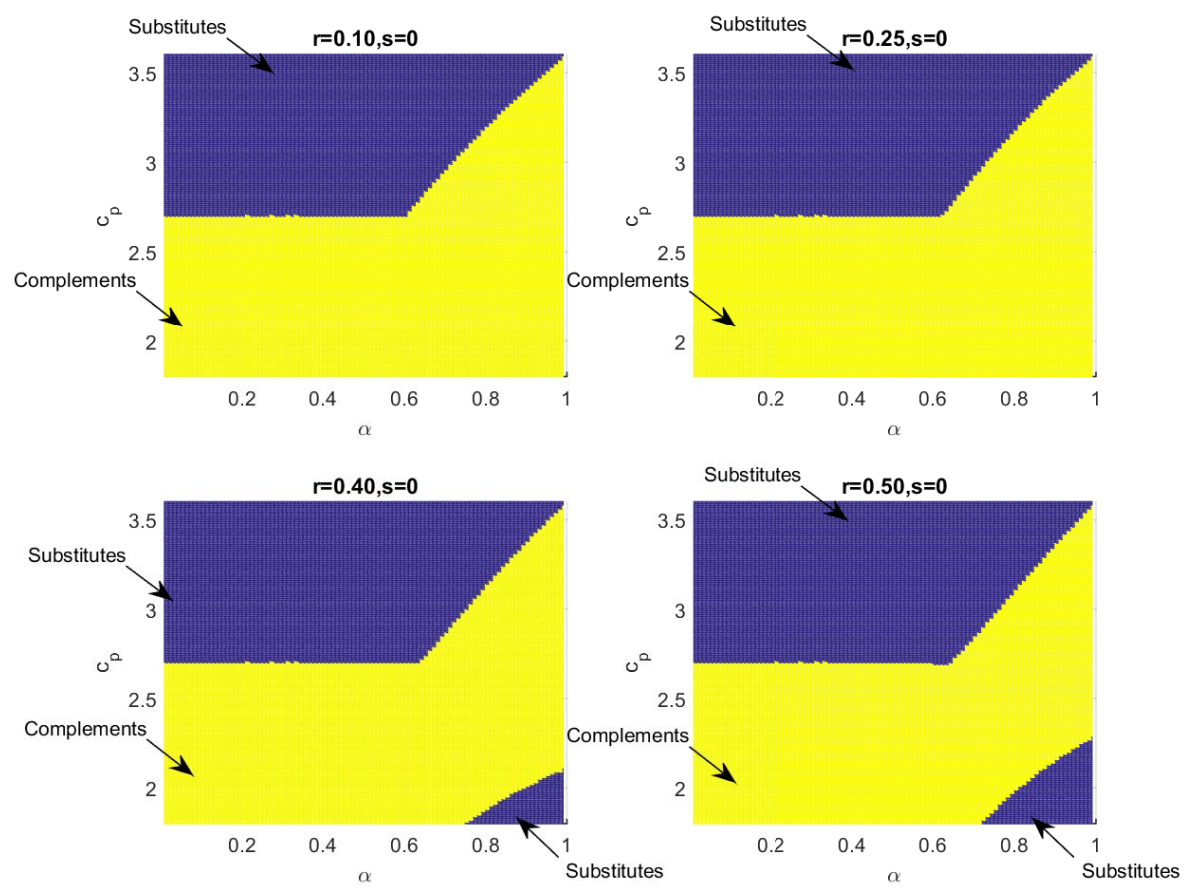

in the basic model. Owing to the analytical complexity involved, we resort to an extensive numerical study to understand how strategic customer behavior affects the interrelationship between demand learning and preference learning.

We present some representative numerical examples to show our analytical results in Figure 5 . To focus on the effect of strategic customer behavior, we let $s=0$ in Figure $5 .{ }^{12}$ We make two interesting observations: First, as customers become more strategic, the region where complementarity occurs becomes smaller. That is, strategic customer behavior weakens the complementarity between demand learning and preference learning. Second, in the presence of strategic customer behavior, when the marginal production cost with customization $c_{p}$ is small and the probability of having high demand $\alpha$ is large, demand learning and preference learning can be substitutes, especially when customers are highly strategic (i.e., $r$ is high), which is in stark contrast to the basic model in which customers are myopic.

We explain the results as follows. The firm does not hurt from strategic customer behavior in three of the four systems, either because it knows the demand exactly and there is no leftover inventory for sale in period 2 (in demand learning and bespoke systems), or the price in period 2

\footnotetext{
${ }^{12}$ We relegate the analysis for the effect of the salvage value $s$ to the Online Supplement.
} 
is so high that customers' waiting incentive is zero (in the preference learning system). However, in the traditional system, strategic customer behavior hurts the firm (numerically confirmed in our setting). Therefore, as customers become more strategic or forward-looking, the region where demand learning and preference learning are complements shrinks. The firm in the traditional system can suffer more from strategic behavior when the probability $\alpha$ of having high demand is large. This is because in this setting, the firm tends to build a high level of inventory in the first period, which implies a potential high level of leftover inventory in period 2 and then a significant waiting incentive for strategic customers. In the preference learning system, knowing that the demand is most likely to be high and the marginal production cost with customization $c_{p}$ is low, the firm produces/orders a high quantity $d_{H}$ (Lemma 4.1). Therefore, the firm in the preference learning system benefits more from a low marginal production cost $c_{p}$ compared to that in the bespoke system where the expected production quantity is $\mu_{D}$ (note that $\mu_{D}<d_{H}$ ). This explains why demand learning and preference learning can be substitutes (i.e., $\pi_{0}^{*}+\pi_{A P}^{*}<\pi_{A}^{*}+\pi_{P}^{*}$ ) for small marginal production cost with customization $c_{p}$ in this setting. In conclusion, strategic customer behavior can significantly dilute the complementarity between demand learning and preference learning, especially when the marginal production cost with customization is low and the probability of having high demand is high.

\subsection{Correlation between Demand and Preference Learnings}

In the basic model, we assumed that the firm learns demand quantity and consumer preference independently through different mechanisms. In practice, sometimes these two kinds of learnings can be correlated. For example, when the firm investigates consumer preference using surveys, it can also have a better idea of how many consumers would like to purchase the product and thus the potential demand. In this section, we investigate the effect of this correlation by analyzing a setting in which the firm can also imperfectly learn the other uncertainty when it learns one of them perfectly.

The results for the traditional system and the bespoke system remain the same as in the basic model, because there is no learning at all in the former and the firm perfectly learns both demand quantity and consumer preference in the latter. We normalize $d_{L}$ to zero for analytical tractability in this section. For expositional convenience, we introduce the notations: $I_{A}\left(\alpha, c_{p}\right) \equiv 1-\frac{w-c_{p}}{(1-\alpha) w}$ and $I_{B}\left(\alpha, c_{p}\right) \equiv 1-\frac{c_{p}}{\alpha w}$. Propositions A-6 and A-7 in the Online Supplement summarize the analytical results for the demand learning system in which consumer preference is also imperfectly learned and for the preference learning system in which demand is also imperfectly learned, respectively. We are able to provide all the necessary and sufficient conditions for demand learning and preference 
learning to be complementary, and we find that the parameters $\alpha$ and $c_{p}$ jointly determine the interrelationship between two learning mechanisms, which is similar to the basic model. However, accuracy levels $I$ and $J$ also play a critical role here, as shown in Proposition 6:

Proposition 6. In the presence of learning correlation, suppose $I \in\left[0, \min \left\{I_{A}\left(\alpha, c_{p}\right), I_{B}\left(\alpha, c_{p}\right)\right\}\right]$ and $J \in\left[0, \min \left\{1-\frac{w-c}{t}, 1-\frac{\alpha(w-c)^{2}}{2(1-\alpha) t c_{p}+\alpha\left(w-\frac{c}{\alpha}\right)^{2}}\right\}\right]$. Then demand learning and preference learning are complements if, and only if, one of the following two conditions holds:

(i) $\alpha \leq \frac{c}{w}$, and $c_{p} \leq w-\frac{(w-c)^{2}}{2 t(1-J)}$.

(ii) $\alpha>\frac{c}{w}$, and $c_{p} \leq \bar{C}_{p}(\alpha, J)$, where $\bar{C}_{p}(\alpha, J) \equiv \max \left\{w-\frac{1}{2 t}\left[\frac{(w-c)^{2}}{1-J}-\left(w-\frac{c}{\alpha}\right)^{2}\right], \alpha w\right\}$.

Proposition 6 shows that with small $I$ and $J$, demand learning and preference learning are complements when $c_{p}$ is small but become substitutes when $c_{p}$ is large. This insight is consistent with that in the basic model. Numerically, we observe that as the learning accuracies $I$ and $J$ increase, the range for complementarity shrinks. This is because with the correlation between the two learning mechanisms, the profit of demand learning (resp., preference learning) system increases as the preference learning accuracy level $J$ (resp., demand learning accuracy level $I$ ) increases - and thus these two learning mechanisms are less likely to be complementary.

\section{Conclusion and Discussion}

Firms in the fashion apparel industry have recently attempted mass customization by launching "Bespoke," a business strategy that allows customers to design and purchase their own and personalized products. At its core, the bespoke strategy combines demand (quantity) learning and preference learning. In this paper, we developed an analytical framework to study the economic value of such a bespoke system and investigate the interaction of demand learning and preference learning when selling to heterogeneous consumers. We found that it is possible for demand learning and preference learning to be either complements or substitutes, depending on the customization/personalization cost and the demand uncertainty profile. They are generally complements when the personalization cost is low and the probability of having high demand is large. Contrary to conventional wisdom, we showed that higher demand uncertainty does not necessarily yield more complementarity benefits. We also found that the complementarity benefit between demand learning and preference learning becomes weaker when customers are more strategic. Interestingly, the substitute loss can occur when the personalization cost is small and the probability of having high demand is large - if customers are strategic.

In our model, the bespoke system requires a "make-to-order" production system which can fulfill demand within a relatively short period of time. However, not all firms have the operational 
capability to complete the whole production process quickly enough in practice. In such a case, firms can use a hybrid system: first build inventories of semi final or modular products based on demand forecast (i.e., make-to-stock), then personalize products based on customer preferences (i.e., maketo-order). We have analyzed such a system and have found that the condition for demand learning and preference learnings' being complements is structurally similar to that in the basic model. ${ }^{13}$

Our theoretical findings have empirically testable implications. The bespoke system is generally attractive when the marginal production cost with customization is low, the probability of having high demand is large, and customers are not highly strategic. A higher fixed investment cost of Bespoke directly undermines its value. We would expect to see most bespoke implementations in precisely these conditions. This has important implications, for the costs of personalization and bespoke practices decrease owing to advanced manufacturing technologies such as 3D printing (Vance 2010). One could collect data on those costs as well as market demand profile. Consumer behavior characteristics might be empirically inferred using structural estimation methods (see Li et al. 2014 for an excellent example). One could then test their relationships with the adoption decisions of bespoke. This presents opportunities for future empirical research.

Acknowledgements: The authors are indebted to the Department Editor Serguei Netessine, the former Department Editor Yossi Aviv, the anonymous Associate Editor, and three anonymous referees for their constructive advice and comments that have substantially improved the paper in both content and exposition. The authors would like to thank Yimin Yu for his contribution in an earlier version of the paper and constructive discussions.

\section{References}

Alptekinoğlu, A., C. J. Corbett. 2008. Mass customization vs. mass production: variety and price competition. Manufacturing and Service Operations Management 10(2) 204-217.

Alptekinoğlu, A., C. J. Corbett. 2010. Leadtime-variety tradeoff in product differentiation. Manufacturing and Service Operations Management 12(4) 569-582.

Athey, S., A. Schmutzler. 1995. Product and process flexibility in an innovative environment. Rand Journal of Economics 26(4) 557-574.

Cachon, G. P., R. Swinney. 2011. The value of fast fashion: Rapid production, enhanced design, and strategic consumer behavior. Management Science 57(4) 778-795.

Chowney, V. 2011. Burberry attempts 'mass customisation' with Burberry Bespoke. Available from http://econsultancy.com/uk/blog/8227-burberry-attempts-mass-customisation-with-burberryBespoke

\footnotetext{
${ }^{13}$ The analysis is available from the authors.
} 
Çil, E. B., M. S. Pangburn. 2017. Mass Customization and Guardrails: "You Can Not Be All Things to All People". Production and Operations Management https://doi.org/10.1111/poms.12716

Cooper, N. 2012. Emerging luxury strategies: Insights from BrandZ. Available from warc.com.

Dobson, G., C. A. Yano. 2002. Product offering, pricing, and make-to-stock/make-to-order decisions with shared capacity. Production and Operations Management 11(3) 293-314.

Fay, S., D. Mitra, Q. Wang. 2009. Ask or infer? Strategic implications of alternative learning approaches in customization. Intern. J. of Research in Marketing 26(2) 136-152

Franke, N., P. Keinz, C. J. Steger. 2009. Testing the value of customization: When do customers really prefer products tailored to their preferences? Journal of Marketing 73 (September) 103-121.

Franke, N., M. Schreier, U. Kaiser. 2010. The "I designed it myself" effect in mass customization. Management Science 56(1) 125-140.

Gaur, V., D. Honhon. 2006. Assortment planning and inventory decisions under a locational choice model. Management Science 52(10) 1528-1543.

Green, P. E., V. Srinivasan. 1978. Conjoint analysis in consumer research: Issues and outlook. Journal of Consumer Research 5(2) 103-23.

Green, P. E., V. Srinivasan. 1990. Conjoint analysis in marketing research: New developments and directions. Journal of Marketing 54(4) 3-19.

Hotelling, H. 1929. Stability in competition. Econ. J. 39 41-57.

Huang, T., J. A. Van Mieghem. 2013. The promise of strategic customer behavior: On the value of click tracking. Production and Operations Management 22(3) 489-502.

Huang, T., J. A. Van Mieghem. 2014. Clickstream data and inventory management: Model and empirical analysis. Production and Operations Management 23(3) 333-347.

Huang, T., Q. Liu. 2015. Strategic capacity management when customers have boundedly rational expectations. Production and Operations Management 24(12) 1852-1869.

Hutzler, K. 2011. Burberry Bespoke trench coats show major shift in brand strategy. Luxury Daily. Available from http://www.luxurydaily.com/burberry-undergoes-major-shift-in-strategy-withBespoke-trench-coats/

Jerath, K., S. Netessine, S. K. Veeraraghavan. 2010. Revenue management with strategic customers: LastMinute selling and opaque selling. Management Science 56(3) 430-448.

Lariviere, M. 2010. Customization and the newsvendor. The Operations Room Blog from Kellogg Insight. Available from http://operationsroom.wordpress.com/2010/05/17/customization-andthe-newsvendor/

Lariviere, M. 2011. Mass customization at Burberry. The Operations Room Blog from Kellogg Insight. Available from http://operationsroom.wordpress.com/2011/11/22/mass-customization-at-burberry/ 
Lee, H. L., C. S. Tang. 1997. Modelling the costs and benefits of delayed product differentiation. Management Science 43(1) 40-53.

Li, J., N. Granados, S. Netessine. 2014. Are consumers strategic? structural estimation from the air-travel industry. Management Science 60(9) 2114-2137.

Li, C., F. Zhang. 2012. Advance demand information, price discrimination, and preorder strategies. Manufacturing Service Oper. Management 15(1) 57-71.

Liang, C., M. Cakanyildirim, S. P. Sethi. 2014. Analysis of product rollover strategies in the presence of strategic customers. Management Science 60(4) 1033-1056.

Liechty, J., V. Ramaswamy, S. H. Cohen. 2001. Choice menus for mass customization: An experimental approach for analyzing customer demand with an application to a Web-based information service. $J$. Marketing Res. 38(2) 183-196.

Mendelson, H., A. K. Parlakturk. 2008a. Product-line competition: customization vs. proliferation. Management Science 54(12) 2039-2053.

Mendelson, H., A. K. Parlakturk. 2008b. Competitive customization. Manufacturing Service Oper. Management 10(3) 377-390.

Milgrom, P., J. Roberts. 1990. The economics of modern manufacturing: Technology, strategy, and organization. Amer. Econom. Rev. 80(3) 511-528.

Murthi, B. P. S., S. Sarkar. 2003. The role of the management sciences in research on personalization. Management Science 49(10) 1344-1362.

Netessine, S., T. A. Taylor. 2007. Product line design and production technology. Marketing Science 26(1) 101-117.

Özer, Ö. 2011. Inventory management: Information, coordination and rationality. Chapter 13 in Handbook of Production Planning, Ed. K. Kempf, P. Keskinocak and R. Uzsoy, 321-365.

Pal, N., A. Rangaswamy. 2003. The Power of One: Gaining Business Value from Personalization Technologies, Trafford, England.

Petruzzi, N. C., M. Dada. 1999. Pricing and the newsvendor problem: A review with extensions. Operations Research 47(2) 183-194.

Pine II, J. B. 1999. Mass Customization. Harvard Business School Press, Cambridge, MA.

Randall, T., C. Terwiesch, K. T. Ulrich. 2007. User design of customized products. Marketing Science 26(2) 268-283.

Rozhon, T. 2004. Worried merchants throw discounts at shoppers. New York Times (December 4), http://www.nytimes.com/ 2004/12/04/business/04markdown.html.

Rice, A. 2010. What's a dress worth? New York Magazine (February 14), http://nymag.com/fashion/10/spring/63807/. 
Shin, H., T. Tunca. 2010. Do firms invest in forecasting efficiently? The effect of competition on demand forecast investments and supply chain coordination. Operations Research 58(6) 1592-1610.

Sonne, P. 2011. Mink or fox? The trench gets complicated, The Wall Street Journal, http://online.wsj.com/article/SB10001424052970203804204577013842801187070.html.

Syam, N. B., N. Kumar. 2006. On customized goods, standard goods, and competition. Marketing Science 25(5) 525-537.

Tereyağoğlu, N., S. Veeraraghavan. 2012. Selling to conspicuous consumers: Pricing, production and sourcing decisions. Management Science 58(12) 2168-2189

Terwiesch, C., C. H. Loch. 2004. Collaborative prototyping and the pricing of custom-designed products. Management Science 50(2) 145-158.

Vance, A. 2010. 3-D printing spurs a manufacturing revolution. New York Times 13.

Wang, T., Toktay B. 2008. Inventory management with advance demand information and flexible delivery. Management Science 54(4) 716-732.

Wang, J., H. Shin. 2015. The impact of contracts and competition on upstream innovation in a supply chain. Production and Operations Management 24(1) 134-146.

Wallace, A. 2010. Putting customers in charge of design. The New York Times. Available from http://www.nytimes.com/2010/05/16/business/16proto.html

Wind, J., A. Rangaswamy. 2001. Customerization: The next revolution in mass customization. J. Interactive Marketing 15(1) 13-32. 